\title{
The Evolution of Sexually Antagonistic Phenotypes
}

\author{
Jennifer C. Perry ${ }^{1,2}$ and Locke Rowe ${ }^{3}$ \\ ${ }^{1}$ Jesus College, University of Oxford, Oxford OX1 3DW, United Kingdom \\ ${ }^{2}$ Edward Grey Institute, Department of Zoology, University of Oxford, Oxford OX1 3PS, United Kingdom \\ ${ }^{3}$ Department of Ecology and Evolutionary Biology, University of Toronto, Toronto, Ontario M5S 3B2, Canada \\ Correspondence: jennifer.perry@zoo.ox.ac.uk
}

Sexual conflict occurs whenever there is sexually antagonistic selection on shared traits. When shared traits result from interactions (e.g., mating rate) and have a different genetic basis in each sex (i.e., interlocus conflict), then sex-specific traits that shift the value of these interaction traits toward the sex-specific optimum will be favored. Male traits can be favored that increase the fitness of their male bearers, but decrease the fitness of interacting females. Likewise, female traits that reduce the costs of interacting with harmful males may simultaneously impose costs on males. If the evolution of these antagonistic traits changes the nature of selection acting on the opposite sex, interesting coevolutionary dynamics will result. Here we examine three current issues in the study of sexually antagonistic interactions: the female side of sexual conflict, the ecological context of sexual conflict, and the strength of evidence for sexually antagonistic coevolution.

\begin{abstract}
Conflict requires some interaction or common activity between males and females... which generates the constraint that the ideal optima for each sex cannot be achieved simultaneously.
\end{abstract}

—Geoff A. Parker 2006, p. 235

$M$ any traits are shared between the sexes, either because they result from an interaction between the sexes or because they share an underlying genetic basis. Examples of the first set may include mating rate, copulation duration, reproductive schedule, fecundity, fertility, and remating rate (Fig. 1A). We can refer to this set of shared traits as interaction traits. They are likely to have different genetic bases in each sex, and therefore can be examples of inter- locus conflict. Examples of the second include most traits that are expressed in both sexes with a common genetic architecture between the sexes, such as human height or pelvis shape. These traits can be examples of intralocus conflict. The evolutionary interests of the two sexes must often diverge because of their different life histories, beginning with anisogamy (Parker 1979; Holland and Rice 1998; Arnqvist and Rowe 2005; Schärer et al. 2012). Therefore, sexual conflict over the value of these shared traits is expected. More specifically, sex specific optima for shared traits yields sexually antagonistic selection on their values (Fig. 1A) (Rowe and Day 2006). Here we will focus on interlocus

Editors: William R. Rice and Sergey Gavrilets

Additional Perspectives on The Genetics and Biology of Sexual Conflict available at www.perspectivesinmedicine.org

Copyright (C) 2015 Cold Spring Harbor Laboratory Press; all rights reserved; doi: 10.1101/cshperspect.a017558

Cite this article as Cold Spring Harb Perspect Biol 2015;7:a017558 
J.C. Perry and L. Rowe

A

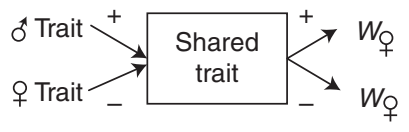

B

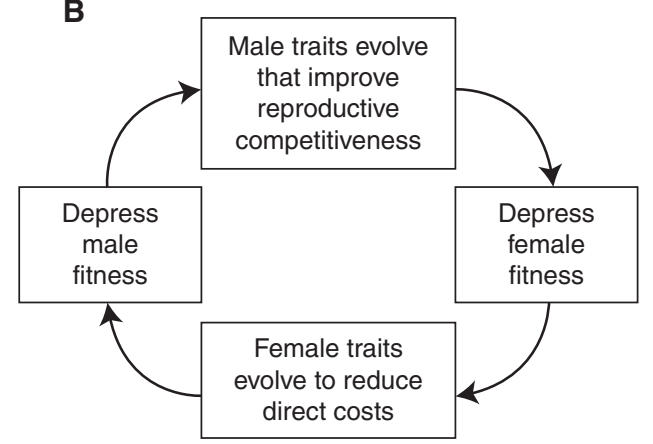

Figure 1. Sexual conflict and sexually antagonistic coevolution. (A) Sexual conflict arises over traits shared by the sexes, in this case traits that are the outcome of sexual interactions that impact fitness in both sexes, and is defined by opposite signs of selection on the trait in each sex. Conflict creates selection on sex-specific traits that shift the value of the interaction traits toward sex-specific optima. This conflict is interlocus because it is expected that the male and female traits involved will have a different genetic basis in each sex. (Adapted from Rowe and Day 2006.) (B) Sexually antagonistic coevolution arises as evolutionary change in each sex alters selection on the other, resulting in further evolutionary change. (Adapted from Arnqvist and Rowe 2005.)

sexual conflict over shared traits resulting from interactions between the sexes.

Interlocus sexual conflict is appealing to evolutionary biologists because it has the potential to be a powerful force driving phenotypic divergence of the sexes. Yet, at the same time, the genetic ties that bind the sexes may act to impede the evolution of each sex toward its optimum. These selective forces and genetic constraints have many additional interesting implications for population demography, reproductive isolation and speciation, and evolutionary diversification, including morphology, physiology, and behavior. The field has moved from an initial period when ideas were first developed mainly by Parker (1979) through a burst of interest sparked by Holland and Rice's (1998) description of chase-away selection, with further development and synthesis in Arnqvist and Rowe's (2005) monograph. By 2006, sexual conflict was declared a new paradigm (Tregenza et al. 2006).

This recent burst of research has not been without controversy, including early disagreements that often centered on defining the scope of the field and how to test its predictions (Chapman et al. 2003a; Cordero and Eberhard 2003; Eberhard and Cordero 2003; Pizzari and Snook 2003; Arnqvist 2004; Eberhard 2004a; Schärer et al. 2012; Ah-King 2013; Kokko et al. 2013), as well as recent critiques evaluating the potential for gender bias in sexual conflict theory and focus (Green and Madjidian 2011; Madjidian and Green 2012; Perry and Rowe 2012a; Dougherty et al. 2013). In recent years, a new era has begun as the field has matured, with studies considering implications for topics like aging (Promislow 2003; Bonduriansky et al. 2008), population structure (Eldakar et al. 2009b; Chang and Sih 2013), kin selection (Rankin 2011; Carazo et al. 2014), human health (Badcock and Crespi 2008; Morrow and Connallon 2013), and extinction (Kokko and Brooks 2003).

A key pathway from sexual conflict over some sexual interaction to diversity is the evolution of sexually antagonistic (SA) traits (Fig. 1A). When an interaction trait-such as mating rate or female remating - is under sexually antagonistic selection, then selection will favor the altered expression of existing traits or the evolution of entirely novel traits that effect a change in mating toward the bearer's optimal mating rate. For example, in a conflict over mating rate (the interaction), male grasping traits that increase mating, and female antigrasping traits that decrease mating (the SA traits) will be favored and may coevolve (Parker 1979; Arnqvist and Rowe 2005). Much progress has been made 
in the study of these traits and the forces shaping their evolution. However, there are still major gaps in understanding some of the most basic aspects of sexual conflict and its influence on SA traits. In fact, many of the questions put forward in initial reviews remain pressing today.

Here, we review current understanding of male and female SA adaptations or armaments. We begin with a brief review of theory for the evolution of sexual armaments and SA coevolution, followed by a survey of male antagonistic adaptations (for a more detailed review, see Arnqvist and Rowe 2005). We then focus on recent advances in three important areas that we believe warrant further study: (a) the female side of sexual conflict; (b) the ecological signature in sexual conflict; and (c) sexually antagonistic coevolution. We discuss in detail four well-known case studies to highlight the diversity of recent advances and remaining questions.

\section{SEXUALLY ANTAGONISTIC COEVOLUTION IN THEORY}

At the heart of sexually antagonistic coevolution (SAC) is the potential for evolutionary change in one sex to modify selection acting on the opposite sex, such that the sexes continuously drive evolutionary change in each other (Fig. 1B). The theory for SAC was substantially worked out by Parker three and a half decades ago (Parker 1979). Subsequent verbal and mathematical models of this process focused primarily on one outcome: escalating arms races characterized by the investment in and exaggeration of sexual armaments in both sexes (Holland and Rice 1998; Gavrilets 2000; Gavrilets et al. 2001; Gavrilets and Waxman 2002; Gavrilets and Hayashi 2006). Further theoretical effort has made it clear that, although escalating arms races are one possible outcome, they are expected only in restricted circumstances or for transitory evolutionary periods (Härdling and Smith 2005; Rowe et al. 2005; Hayashi et al. 2007). Both earlier and later models show that a range of alternative forms of coevolutionary outcomes are possible, including stable equilibria, coevolutionary cycles, and de-escalation of armaments in both sexes.
These theoretical analyses show that the dynamics and outcome of SAC depend on (1) the form of female resistance, (2) the economics of mating and how selection acts on females in particular, and (3) population-specific factors, including local ecology and initial trait values.

\section{The Form of Female Resistance}

Models of SAC are often set up similarly to traditional models of sexual selection, in which there is a male trait and a female response (or preference), which interact to determine mating, with potential impacts for fitness in both sexes. In SAC models, there is an antagonistic sexually selected male trait (i.e., whose expression decreases female fitness) and a female antagonistic trait that determines female responses to that male trait. These interact to determine the value of the shared interaction trait (e.g., mating rate) over which sexual conflict occurs, and determine the extent of SA trait evolution (e.g., Gavrilets et al. 2001; Kokko et al. 2003; Rowe and Day 2006). Some models permit females to evolve resistance to harmful male traits only by escalating their defenses against males through an increased threshold of response to antagonistic male traits. In this scenario, escalating arms races are commonly expected, because males face strong selection favoring the exaggeration of SA traits that overcome female thresholds. However, females might alternatively evolve resistance through decreased sensitivity to harmful male traits, equivalent to a decreased slope for the relationship between female responses and male traits. When female sensitivity is free to evolve, models predict that arms races are much less likely, with other outcomes including the de-escalation of armaments in both sexes, and the evolution of female insensitivity to male traits (Rowe et al. 2005).

These models tell us that an important part of understanding SAC is characterizing the form of the female response function, as determined by female antagonistic traits, and variation in this function (Rowe et al. 2005). Specifically, we need to address the following questions. What is the genetic variation underlying female response functions to male persistence traits? 
Are female responses free to evolve in any direction, or constrained-by a lack of genetic variation or by natural selection acting on female responses-to evolve in certain directions? Do different categories of female resistance traits differ in their evolvability?

\section{The Economics of Mating}

The economy of mating - the costs and benefits of mating for both sexes-determines the extent of conflict and therefore the dynamics of SAC (Arnqvist and Rowe 2005; Chapman 2006; Fricke et al. 2009). Of course, where increased mating is beneficial for both sexes, there will be no conflict over mating rate. But where there is conflict, recent models point to details about the economics of mating that should have a strong influence, and suggest that the economics of mating for females is particularly significant. For example, the relationship between the number of mates and mating costs is potentially important, especially whether every mating beyond the first mating is costly for females or there is an intermediate optimum number of matings (Arnqvist and Nilsson 2000; Kimura and Ihara 2009). If the latter, then the expected coevolutionary outcome varies with the initial state of the system, setting the stage for idiosyncratic outcomes. Furthermore, whether mating costs are fixed or increase with the degree of exaggeration of male sexually antagonistic traits matters, with the latter much less likely to lead to extreme exaggeration in harmful male traits (Parker 1979; see also Kazancioğlu and Alonzo 2012). One can imagine species in which both forms of mating costs occur: fixed per-mating costs are likely in water striders, in which mating costs are imposed externally by predators (Rowe 1994), whereas in seed beetles with spiny male genitalia, increasing spininess results in higher mating costs (Hotzy and Arnqvist 2009). In another example, Härdling and Smith (2005) showed that arms races are much less likely when costs depend on the interaction between the sexes (e.g., when costs arise from behavioral interactions) rather than when costs are fixed independent of interactions (e.g., when costs arise from investing in armament production).
These theoretical predictions prescribe a research program for sexual conflict that is based on measuring the economics of sexual interactions, particularly in females, a point we develop below.

\section{Predictable or Stochastic?}

Models have examined ways in which ecological conditions can determine the outcome of conflict. For example, when higher population density leads to more frequent sexual interactions, the intensity of sexual arms races is expected to increase (Kimura and Ihara 2009). Furthermore, ecological and population factors can promote assortative mating among males and females possessing different SA traits, and this kind of nonrandom mating can maintain intrapopulation variation in SA traits (Härdling and Bergsten 2006). However, this ecologically based predictability stands in contrast to other theoretical expectations that the outcome of SAC should vary with initial conditions and that stochastic effects, including genetic drift, should play a major role (Gavrilets 2000; Hayashi et al. 2007; Kimura and Ihara 2009). These expectations begin with classic models predicting that populations will move by genetic drift along a line of equilibrium on which SA traits are balanced between the sexes (Lande 1981; Gavrilets 2000). The potential importance of ecological variation means that it is vital to investigate the context-dependence of the economics of mating (Fricke et al. 2009; and below).

\section{MALE SEXUAL ARMAMENTS}

Male SA adaptations_ “ "persistence" traits — are defined by their harmful effects on females that interact with males bearing the traits (Figs. 1 and 2). We therefore need measurements of costs in females to identify male harmful traits. In some cases, costs to females are so obvious that measuring them would be superfluous. But even when costs seem obvious - surely the hypodermic aedeagus of bed bugs must impose costs on females? - the evolution of female SA traits like the paragenitalia that mitigate costs can mean that male traits that were once harm- 

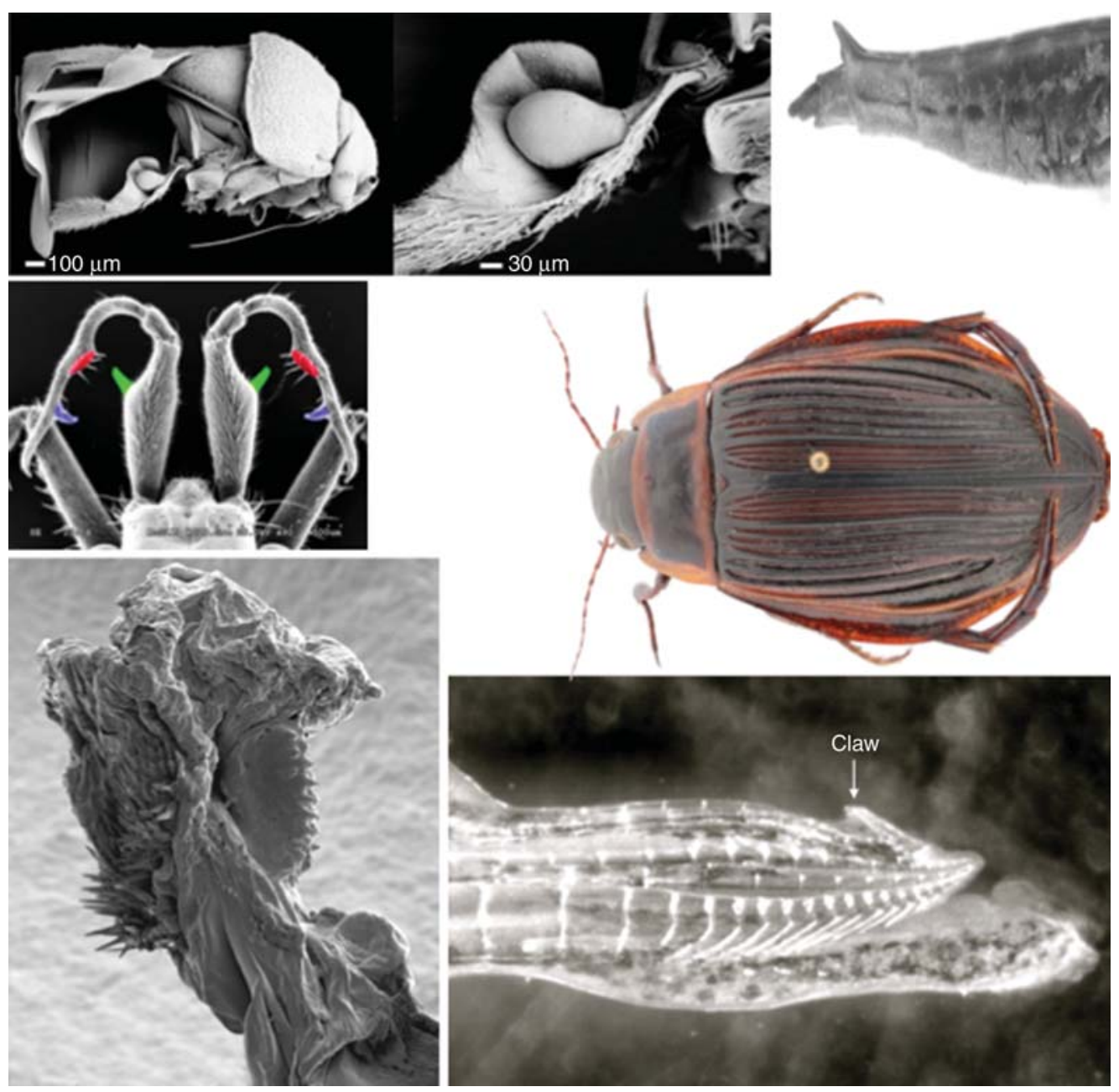

Figure 2. Examples of putatively or well-supported SA traits in males and females. Clockwise from top left: female paragenitalia in the plant bug Coridromius bulbopella, close-up and whole-organism views; the female abdominal spine of the water strider Gerris incognitus; the furrowed antigrasping dorsal surface of a female diving beetle (Dytiscus latissimus); the claw of the male intromittent organ in guppies (Poecilia reticulate); the spines and jawlike structure of the male aedeagus in the seed beetle Callosobruchus subinnotatus; the antennae of male water striders (Rheumatobates rileyi), colored to illustrate several grasping adaptations. (Photographs provided by N. Tatarnic, G. Arnqvist, J. Bergsten, L. Kwan, and A. Khila.)

ful may no longer be so, or the harm may be very much reduced.

These costs to females could arise either from their impact on interaction traits over which there is conflict (by shifting these traits away from the female optimum), or from direct harm to the females themselves. Harm to females can arise as a byproduct of selection on male traits in another context, such as male- male competition. As examples, longer genital spines in male seed beetles cause both increased male success in sperm competition and increased harm to females (Hotzy and Arnqvist 2009; Hotzy et al. 2012; and one allele of a male reproductive gene in Drosophila melanogaster is associated with both decreased female remating rate and decreased female longevity (Fiumera et al. 2006). Alternatively, harm to females can 
in theory be directly favored by selection (Johnstone and Keller 2000), although this prediction has yet to receive empirical support (e.g., Morrow et al. 2003).

Male antagonistic traits and their impacts are much better understood than those of females. This might be owing to the apparently greater frequency in males of morphological SA traits, which can be observed and measured with relative ease compared to behavioral and physiological traits. Classes of persistence traits include grasping appendages, ranging from the adhesive tarsae of diving beetles (Bergsten et al. 2001) to the enlarged and spined and hooked antennae of water striders (Rowe et al. 2006); spiny stabbing genitalia in seed beetles (Crudgington and Siva-Jothy 2000), garter snakes (Friesen et al. 2014), guppies (Fig. 2) (Kwan et al. 2013) and traumatically inseminating bugs (Stutt and Siva-Jothy 2001; Tatarnic et al. 2006); chemical traits, such as seminal proteins that influence female physiology and behavior (e.g., by boosting fecundity or delaying remating beyond the female optimum; Perry et al. 2013), and behavioral traits, such as the harassment of females by males observed in many species. Eberhard (2004a,b, 2010) has suggested that physiological and chemical male SA traits might result in arms races more commonly than morphological or behavioral traits, in part because physiological and chemical traits might be more complex and difficult for females to avoid. At this point, there are not enough data available to evaluate this hypothesis.

In many cases, costs for females are plausible but not straightforward. A prime example is the short-term boost in egg deposition or production after mating, caused by male seminal proteins in many insects (Vahed 2007; Chapman 2008; Perry et al. 2013). Such gonadotropic effects are often interpreted as harmful to females (e.g., Parker and Simmons 1989; Simmons and Parker 1989). But without careful measurement, it is not clear what fecundity stimulation means for female fitness. An increased reproductive rate is generally favored, but increased reproductive effort might entail costs to other life history components. A similar example is the chemical inhibition of female-remating rate by male seminal proteins in many arthropods. This effect is often taken as either male manipulation of female remating rate (e.g., Arnqvist and Nilsson 2000; Wolfner 2002; Arnqvist and Rowe 2005; Crudgington et al. 2005), or evidence of sexual cooperation (Dickson 2008). In fact, there is typically scant evidence for what optimal female remating rates are in natural conditions (Gwynne 2008), largely because measuring this optimum is empirically challenging (Maklakov and Arnqvist 2009).

SA selection and coevolution are expected to drive the rapid diversification of male SA traits. This prediction corresponds well with the substantial phenotypic diversity of male persistence traits, both among populations and species. As examples, both water striders and diving beetles show striking diversity across both populations and species in male grasping armaments (Bergsten et al. 2001; Bergsten and Miller 2007; Khila et al. 2012; Perry and Rowe 2012b), as do traumatically inseminating bug species in their stabbing aedeagus (Tatarnic and Cassis 2010), and there is population-level variation in the effects of male seminal substances on female fecundity and longevity in adzuki bean beetles (Yamane 2013). This phenotypic diversity is matched by the substantial standing genetic variation in male SA traits found in several experimental evolution studies. For example, the genital spines of Callosobruchus beetles respond to artificial selection for increased length within only a few generations (Hotzy et al. 2012). In D. melanogaster, the overall level of male harmfulness to females increases when females are prevented from coevolving with males (Rice 1996; but see Jiang et al. 2011), as well as in response to the experimental manipulation of polyandry (Holland and Rice 1999; Pitnick et al. 2001) and sex ratio (Nandy et al. 2013; but see Wigby and Chapman 2004).

Although there has been much progress, there is much to learn about harmful male traits. We know much more about conditiondependence in sexually selected ornaments and weapons than in SA armaments. We know little about the hypothesis that coevolution between the sexes should result in multiple harmful male 
traits, as females evolve resistance to each in succession (Arnqvist and Rowe 2005). It is intriguing to speculate that this kind of process can account for the diversity and redundancy of male seminal proteins in animal like fruit flies, but evidence is lacking (Arnqvist and Rowe 2005).

\section{SEXUALLY ANTAGONISTIC TRAITS IN FEMALES}

Numerous reviews have called for increased study of the female side of sexual conflict (Zeh and Zeh 2003; Arnqvist and Rowe 2005; Arnqvist 2006; Chapman 2006; Fricke et al. 2009). These reviews highlight the paucity of strong evidence for SA traits in females, and the very limited data available on forms of resistance, natural selection on female SA traits, and their genetic variability and evolvability. What progress has been made?

In short, there remains only limited evidence identifying female SA traits. To establish that a female trait is sexually antagonistic, we need to work out the relationships depicted in Figure 1A. We need to show SA selection acting on a trait that is the outcome of sexual interactions, and to show that a putative female SA trait impacts the interaction trait in a way that improves female fitness. The most convincing demonstrations of female resistance adaptations are from studies that identify an interaction trait that negatively impacts female fitness. The most common example is mating rate, which is often costly for females beyond some intermediate value. Studies that experimentally manipulate the interaction trait to evaluate the impact on female fitness are most valuable in establishing causality.

Measuring the relationship between interaction traits and female fitness is not trivial. A major difficulty is that we would like to have data from natural conditions, but for many organisms, natural mating behavior and the range of ecological settings is unknown or highly variable. A further difficulty is that for many species it is difficult to engineer phenotypes that are costly for either sex, because organisms have evolved strategies to avoid these phenotypes.
Hence, it is easy to experimentally reduce mating rate, but difficult to increase it because females resist excess mating. Furthermore, we would ideally like to have data that describe variation in female lifetime reproductive success, rather than components of fitness (Maklakov and Arnqvist 2009). This is because the potential for trade-offs among life history components greatly complicates extrapolating fitness components to total fitness. For example, a negative effect of mating rate on female longevity is sometimes portrayed as a cost of mating, but may represent an adaptive shift in female life history. When this is the case, decreased longevity is not a cost imposed by males but by allocation tradeoffs between investment in reproductive effort and somatic maintenance. Complicating things further, it is clear that male SA traits can impact not just offspring number but also quality (Gasparini et al. 2012) —and possibly grandoffspring quality (Brommer et al. 2012).

\section{Types and Forms of Female Resistance} Traits

Comprehensive surveys in arthropods suggest that female SA traits might frequently be behavioral or physiological rather than morphological (e.g., Eberhard 1997; Eberhard 2004b). Alternatively, it may be that it is difficult to assess the evolutionary history and current function of female morphological traits from measurements made on static specimens. For example, grooves and slots in female genitalia that appear to be cooperative guides for male genitalia (Eberhard 2004b) might have evolved to minimize male-imposed costs. The spermalege of female bed bugs appears to have evolved to minimize costs from the hypodermic aedeagus of male bed bugs (Morrow and Arnqvist 2003). In any case, behavioral resistance seems likely when the economics of mating are very context-dependent, such that females need to finetune resistance to local conditions.

We have emphasized above the theoretical prediction that the form of female resistancein terms of the threshold and sensitivity of female responses to male traits (Fig. 3) — should 
J.C. Perry and L. Rowe
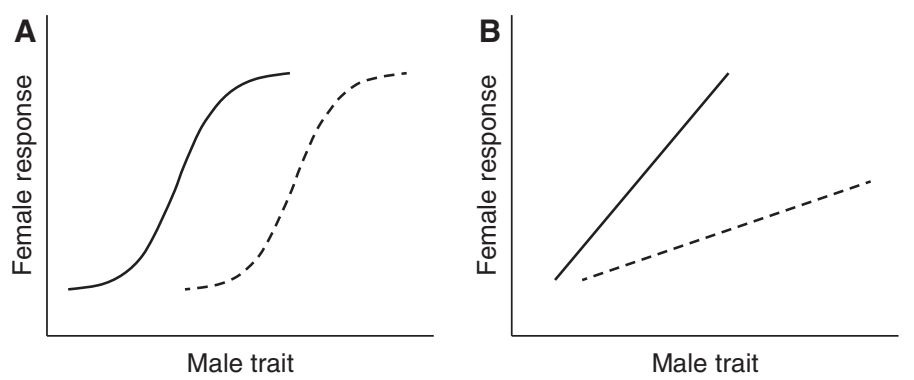

Figure 3. Female responses to male sexually antagonistic traits can take diverse forms. Increased female resistance to male traits is illustrated by a shift from the solid line to the dashed line within each panel, and can take the form of $(A)$ an increased threshold of response, or $(B)$ decreased sensitivity.

have significant impact on the outcome of SAC. We therefore need data that characterize which forms of female response are evolutionary labile. How does natural selection act on female SA traits? How much additive genetic variation do they display, and in which directions? This remains a significant gap, and there are almost no data available on this topic, although studies of genetic variation in female resistance are now being conducted.

\section{Genetic Variation for Resistance to Harm}

Several studies in D. melanogaster have detected additive genetic variation in female ability to resist harm from males (e.g., Wigby and Chapman 2004; Linder and Rice 2005; Lew et al. 2006; Brommer et al. 2012). However, functional understanding is often lacking, and in general it is not known what specific SA traits in females mediate this variation. Instead, the data generally come from studies of the genetic variation underlying female fecundity, sometimes along with longevity, in response to male harm. There are exceptions; for example, one study of $D$. melanogaster was able to link female reluctance to remate with increased resistance to harm from males (Lew et al. 2006). Another potential example comes from work on the nuptial feeding bushcricket Gryllodes sigillatus, in which there is genetic variation in the duration females feed on spermatophylax nuptial gifts from males (Gershman et al. 2013); these gifts are thought to manipulate female physiology in potentially costly ways for females.

\section{THE CONDITIONAL ECONOMICS OF CONFLICT}

We have previously argued that we need a much more ecological understanding of sexual conflict because ecological context determines the economics of conflict (Arnqvist and Rowe 2005; Fricke et al. 2009). The reasons for this are threefold. First, the ecological context of sexual interactions will often determine whether there is conflict or congruence, because costs and benefits to each sex depend on ecological context (Arnqvist and Rowe 2005). Moreover, the extent of conflict may vary among individuals within a population depending on their condition. For example, in D. melanogaster, males cause the greatest harm to females of highest fecundity (Lew et al. 2006; Long et al. 2009) and large males cause greater harm to females than small males (Friberg and Arnqvist 2003). Second, ecological context can determine the extent of natural selection on SA traits. For example, in water striders, antagonistic abdominal spines in females increase susceptibility to cannibalism and predation during molt (Arnqvist 1994). Therefore, natural selection against these spines will be a function of local density of conspecifics and predators. Third, both theoretical and empirical studies have shown that ecological context can determine the direction and extent of SAC (e.g., Martin and Hosken 2003; Rowe and Day 2006; Fricke et al. 2010a; Arbuthnott et al. 2014). 
An excellent example of the importance of ecological context is the sex peptide of D. melanogaster, often considered a prime example of an SA male trait (Arnqvist and Rowe 2005; Lessells 2006; Rowe and Day 2006). Females experience strong postmating changes in behavior from receipt of sex peptide, including increased egg-laying in the short term and delayed remating, among a suite of other changes (Chapman et al. 2003b; Fricke et al. 2013). Sex peptide appears to decrease female fitness in some contexts. In response to receipt of sex peptide, females achieve lower lifetime reproductive success (Wigby and Chapman 2005). These and related results have sparked a great deal of interest in this protein. However, the magnitude and even the existence of costs for females depend strongly on nutrient levels (Rogina 2009; Fricke et al. 2010b). In fact, receipt of sex peptide permits females to lay more eggs under most nutrient levels, and it is only under extreme nutrient restriction that costs have been reported in two subsequent studies (Rogina 2009; Fricke et al. 2010b). Thus, depending on local food abundance, sex peptide could be considered a prime example of sexual conflict or sexual congruence.

A second intriguing example of the potential importance of ecological context for the evolution of antagonistic traits comes from a recent study of independently evolving D. melanogaster populations (Arbuthnott et al. 2014). Here, replicate populations evolved in one of two novel environments-food supplemented with cadmium or ethanol-and were then contrasted for male harm and female resistance. The investigators found suggestive evidence for ecologically mediated parallel evolution of both male harm and female resistance to that harm. Males evolved on ethanol-supplemented food depressed the lifespan of standard (i.e., not experimentally evolved) females more than did males evolved on cadmium-supplemented food; females evolved on ethanol-supplemented food had a smaller reduction in lifespan following high exposure to males compared with females evolved on cadmium-supplemented food. Depression of female lifespan might represent harm to females-and therefore sexual conflict_or a shift in life history that might have positive or neutral effects on female fitness. If the changes in lifespan do reflect harm, it would be intriguing to investigate the antagonistic traits that underlay harm and resistance to determine whether populations differed in suites of antagonistic traits or simply in the degree of expression of traits common to both sets of populations. However, the study does suggest that ecological conditions can substantially influence the course of evolution of traits involved in sexual conflict.

\section{SEXUALLY ANTAGONISTIC COEVOLUTION}

The limited evidence for female resistance traits inevitably means that there are few systems for which SAC has been well characterized. To our knowledge, these systems include water striders, diving beetles, traumatically inseminating bugs, and seed beetles, each of which is discussed in some detail below. Despite the paucity of examples, the potential for sexual coevolution is one of the most intriguing aspects of sexual conflict. For example, how often does conflict lead to coevolution, diversification, and speciation? There is suggestive and mixed evidence for all of these larger scale outcomes (Arnqvist et al. 2000; Martin and Hosken 2004; Rice et al. 2005; but see Plesnar-Bielak et al. 2013). Even smaller scale questions remain open. For example, as some have argued, does SAC necessarily lead to increased complexity in sexual armaments (Alexander et al. 1997; Eberhard 2004a, 2006; Rowe and Arnqvist 2012)? These questions must remain unanswered until we have more than the small handful of case studies now available. Below we review these case studies.

\section{CASE STUDIES}

\section{Water Striders}

Water striders remain among the best-characterized system for interlocus sexual conflict over sexual interactions. In this group, the sexes are in conflict over mating rate, which is mediated by social and ecological conditions. Several antagonistic traits in both sexes affect mating 
rate, and there is evidence of arms races between these traits (Arnqvist and Rowe 2005). There are quantitative data on the economics and ecology of conflict (Rowe et al. 1994), as well as the trajectory of SAC (Arnqvist and Rowe 2002a,b; Rowe and Arnqvist 2002). Since conflict in the group was last reviewed (Arnqvist and Rowe 2005), there have been several new insights into the behavioral context of conflict, antagonistic traits involved in the conflict, and their coevolution.

Most of the attention on female SA traits in water striders has been directed toward female resistance behavior and pregenital spines (Fig. 2 ), both of which vary among populations and species and affect mating outcomes (Arnqvist and Rowe 2005; Ronkainen et al. 2005; Perry and Rowe 2012b). Han and Jablonski (2009) have identified a new trait in females that may have a substantial effect on SAC. The basal state in Gerris spp. is an exposed vulvar opening, into which males may be able to insert their genitalia without female cooperation, although this has not been shown. In contrast, the pregenital segment 7 of $G$. gracilicornis largely covers the vulvar opening. Han and Jablonski (2009) have suggested that this precludes so-called forced copulation because females must inflate their genitalia to allow male access. An interesting hypothesis is that males have responded by making resistance (delay in inflating genitalia) costly to females, potentially through a "war of attrition"-type process. Han and Jablonski (2010) report that males in this species tap the water surface during the precopula stage, which attracts predators; females respond by inflating their genitalia.

One of the more dramatic grasping traits of male water striders is the enlarged, hooked, and spined male antennae in the genus Rheumatobates (Fig. 2) (Rowe et al. 2006). The fine-scale function and development of these SA traits in Rheumatobates rileyi have recently been reported by Khila et al. (2012). Of the several traits that make up the antennae, all seem finely crafted to match the grasping sites of resistant females during premating struggles. One interesting question is whether this pattern extends across the diverse range of antennal traits and shapes in the group. An apparent lack of morphological diversity in females corresponding to male morphology suggests that this may not be the case (Rowe et al. 2006). Alternatively, there may be many other finely crafted means to grasp females. In a second part of the study, Khila et al. (2012) used RNA interference to show a role for the gene distal-less $(\mathrm{dll})$ in generating the grasping antennal traits of $R$. rileyi. A graded reduction of the grasping traits with knocked down dll expression resulted in a similarly graded reduction of mating success in males. These data suggest continuous selection for elaboration from a point close to the basal state for antennae in this genus.

In Gerris spp., male grasping traits include pregenital and genital segments, which are inflated and curled around the female genitalia. A recent comparative analysis of shape and complexity of these grasping segments suggests that they are evolving antagonistically with their counterparts in females (Rowe and Arnqvist 2012). Moreover, they are more complex and evolving more divergently than similar body parts that do not seem to be involved in sexual conflict. An exception to this is the terminal components of the genitalia, which are particularly complex and divergent, and do not appear to be involved in grasping, but do appear to be under sexual selection (Rowe and Arnqvist 2012). These data on both components of the genitalia provide quantitative evidence for the long-held hypothesis that genitalia are particularly complex and divergent in shape (Eberhard 1985).

The general pattern of SAC in the water strider genus Gerris is one of matched escalation and de-escalation of male grasping traits and female antigrasping traits (Arnqvist and Rowe 2002a,b). When these traits veer from matching they affect mating behaviors; when males are relatively armed mating rates go up, and when females become relatively armed, mating rate goes down (Arnqvist and Rowe 2002a). The connection of these coevolutionary dynamics at the species level to the well-known ecological effects on conflict is not well understood (Rowe and Arnqvist 2002). A recent study of SAC at the population level may be a step toward connect- 
ing these two scales. Perry and Rowe (2012b) report a pattern of coevolution in grasping and antigrasping traits, and mating behaviors at the population level that matches that reported at the species level. A similar study in a closely related species, Gerris gillettei, reported evidence of correlated evolution between the sexes in potentially antagonistic sexual traits (Gagnon and Turgeon 2011). However, in contrast to interspecific studies of Gerris (Rowe and Arnqvist 2002; Arnqvist and Rowe 2002b) and the interpopulation study of Perry and Rowe (2012b), the evolution of female spine size was not correlated to male genital traits. The next step in these interpopulation studies will be to directly examine local ecological (and social) conditions and attempt to connect them to the state of SA traits in the populations.

The social context of sexual conflict has received considerable recent attention in the stream water strider Aquarius spp. In the typical environment of pools connected by flowing water, individuals in different pools often experience different social environments because group composition varies (Sih and Watters 2005). Individual males tend to vary in their aggressiveness to females (and males), which may affect both their own mating fitness and the mating rate of the group (Eldakar et al. 2009a,b; Chang and Sih 2013). The multilevel nature of selection in this system sets up the potential for selection to work in opposition between levels. Eldakar et al. (2009a,b) has argued for a "tragedy of the commons" process in Aquarius remigis. Aggressive or hyperaggressive males tend to harass females at an extremely high frequency in an attempt to mate. They will also harass males and mating pairs. In some conditions, this results in increased relative mating success for these males, but reduced activity of other males (Eldakar et al. 2009a,b; Chang and Sih 2013). Females tend to disperse from pools containing very aggressive males, resulting in a reduction in average mating frequency in the original pool. A very interesting possibility is that male aggression may reduce female fecundity in addition to mating frequen$\mathrm{cy}$, which would result in a tragedy of the commons over the resource (female ova) over which males compete. This hypothesis remains to be tested, but establishing a link between heightened male aggression and decreased female fecundity would provide strong evidence for a sexually antagonistic tragedy of the commons.

\section{Traumatic Insemination in Bugs}

Traumatic insemination - whereby males transfer ejaculates by piercing the female body cavity with syringe-like intromittent organs-has evolved independently in a variety of invertebrate taxa (reviewed by Lange et al. 2013; Tatarnic et al. 2014). The potential for sexual conflict in these systems is clear. Females face increased risk of infection and the costs of mounting an immune response, and these kinds of costs have been detected in a well-studied system of traumatic insemination, Cimex bed bugs (Stutt and Siva-Jothy 2001). Females in these and many other species have evolved remarkable morphological counteradaptations that mitigate the costs of hypodermic insemination, in the form of a unique structure called the spermalege (Fig. 2) (Reinhardt et al. 2003). The spermalege is, in many species, effectively a secondary female genitalic structure, typically consisting of a modified abdominal wall and an internal pocket where injected ejaculate is stored, with wide variation in the presence and form of these structures across bed bug species (Usinger 1966). Evidence that the spermalege is effective in reducing mating costs comes from functional studies in which artificial piercing and insemination within the spermalege region does not harm females, but the same action outside this region is very harmful and often fatal (Morrow and Arnqvist 2003; Reinhardt et al. 2003).

What has previously been lacking in understanding SAC for traumatic insemination and spermalege evolution is formal comparative analyses linking divergence in male and female morphology (Arnqvist and Rowe 2005). A recent study has done just this for traumatically inseminating plant bugs of the genus Coridromius (Tatarnic and Cassis 2010). In these species, male piercing genitalia varies extensively in size and shape; likewise females display a great 
deal of variation in spermalege morphology (Tatarnic et al. 2006). Across the genus, the diversification of male genital morphology and female spermalege morphology are closely correlated. Notably, there are exceptions. In some species, male morphology has diverged with little corresponding evolution in female traits. Functional studies in these species would be most interesting to investigate whether males have gained an advantage in increasing mating rate, overcoming female resistance, transferring more ejaculate, or whether female resistance has taken other forms (e.g., behavioral or physiological resistance).

\section{Seed Beetles}

Seed beetles, Callosobruchus, are renowned for their highly elaborated genitalia that damage female reproductive tracts (Fig. 2). Females resist mating by kicking to dislodge mating males. Crudgington and Siva-Jothy (2000) experimentally ablated female legs so that resistance was prevented. The effect of this was an increase in both mating duration and damage to females (see also Maklakov and Arnqvist 2009). Since these early studies, research has focused on the function of genital spines and on coevolution between male spines and the female reproductive tract.

In a comparative study of populations that varied in genital morphology, Hotzy and Arnqvist (2009) have shown a role for genital spines in sperm competition success (when males were last to mate with a female). Males from populations with longer spines had higher success in sperm competition and this effect remained after controlling for body size variation. In the same study, there was no correlation between harm to the female reproductive tract and male spine length. Two possible nonexclusive roles for the spines in sperm competition are that spines may act as a grasping device to prolong mating or aid in positioning (Edvardsson and Tregenza 2005), or alternatively, scarring of the female tract by the spines may facilitate uptake of accessory substances transferred by the male. Results of the Hotzy and Arnqvist (2009) study support the first hypothesis, as there was no independent effect of scarring on the outcome of sperm competition, and Hotzy and colleagues (2012) report increased female uptake of male ejaculate proteins following matings with males with longer spines.

One might expect an evolutionary response of females to the harm caused to them by spined male genitalia. In comparative study at the species level, Rönn et al. (2007) found a positive correlation across species between spine elaboration and the amount of connective tissue in the copulatory duct, where scarring occurs. These data provide a rare case in which there is strong support for the correlated evolution of a male persistence trait and a female response. It is argued that the male trait is antagonistic because its advantage to males comes at a cost to females. The thickening of the reproductive tract in females seems likely to act as a defensive trait in females; however, it is not yet clear how female reproductive tract thickness affects selection on the male trait. Male spine elaborations may be caused by some other aspect of female morphology or behavior, or caused entirely by environmental variables that differ between species. Therefore, it is not obvious that these data support a case of SA coevolution.

\section{Diving Beetles}

Diving beetles (Dytiscidae) provide one of the few cases in which we have strong evidence of sexual coevolution in nature that appears to be antagonistic. Males chase and grasp females in an attempt to mate and females attempt to dislodge males with repeated dives and erratic swimming. A cost of mating to females has not been quantified, but a lack of access to air (they are air breathers) has been suggested (Bergsten and Miller 2007). Males grasp females on the pronotum and elytra with tarsae modified to include a set of suction cups. The females of some species and populations have evolved rough dorsal surfaces that appear to impede grasping (Fig. 2). In an early study of Graphoderus zonatus verrucifer, Bergsten et al. (2001) provided evidence of a correlation between the expression of male grasping traits and the frequency of the rough dorsal surfaced female. This 
was the first evidence pointing to SAC at the population level.

Two key pieces of the story that were previously missing have now been addressed. First, the utility of the male suction cups in grasping females, and the obstruction of grasping by the rough females surface, had not been shown. A recent biomechanical study supports these hypotheses (Green et al. 2013). Moreover, the work indicated an interaction that suggests that males with tarsae suited to grasping rough surfaced females were unsuited to grasping smooth surfaced females. This interaction implies a tradeoff, which would play into the correlated evolution of male and female features. Second, recent comparative analysis provides strong evidence for the correlated evolution of male and female structures at the species level (Bergsten and Miller 2007). The combination of known traits and correlated evolution at the both the population and species level makes this a very interesting group for intense study. A necessary step for further study of this group as a model for SAC is an understanding of the economics of mating.

\section{CONCLUSIONS}

Theoretical analysis points to the importance of the form of female resistance and the forces of selection acting on it. These analyses mirror those in more traditional sexual selection models (Kirkpatrick and Ryan 1991). Despite this, there are precious few identified SA traits in females_-most of which are behavioral-and fewer still where their role in antagonistic coevolution has been studied. Therefore, we continue the call for further study of female SA traits and their evolutionary dynamics (Zeh and Zeh 2003; Rowe et al. 2005; Arnqvist 2006; Fricke et al. 2009; Findlay and Swanson 2010; Perry and Rowe 2012a).

Models also illustrate that the course of SAC is determined to a large degree by natural selection acting on both interaction traits and SA traits in the two sexes. There is very limited data here, particularly on SA traits. One reason to understand these forces of selection is to enable predictions about how the ecological context will determine both the extent of conflict and the course of SAC (Rice et al. 2006; Rowe and Day 2006). This means that we need to understand the economy of conflict in natural systems. Even in some of the better-known systems (e.g., water striders, diving beetles), the connection between the ecological context of conflict and the course of SAC is unknown.

Finally, the number of demonstrations of SAC remains a handful and it is dominated by studies in insects. More species of a broader taxonomic set (e.g., King et al. 2013; see works by Schärer et al. 2014 and Schneider, 2014) will allow us to assess the importance of SAC in driving the evolution of diversity, and will almost certainly reveal entirely novel processes.

\section{ACKNOWLEDGMENTS}

We thank S. Gavrilets and W. Rice for the invitation to contribute to this collection, and G. Arnqvist, W. Rice, and an anonymous reviewer for helpful comments that improved the manuscript. We thank those who kindly provided the images of Figure 2. J.C.P. is supported by Jesus College (Oxford). L.R. is supported by the Natural Sciences and Engineering Research Council and the Canada Research Chairs program.

\section{REFERENCES}

* Reference is also in this collection.

Ah-King M. 2013. On anisogamy and the evolution of "sex roles." Trends Ecol Evol 28: 1-2.

Alexander RD, Marshall DC, Cooley JR. 1997. Evolutionary perspectives on insect mating. In The evolution of mating systems in insects and arachnids (ed. Choe JC, Crespi BJ), pp. 4-31. Cambridge University Press, Cambridge.

Arbuthnott D, Dutton EM, Agrawal AF, Rundle HD. 2014. The ecology of sexual conflict: Ecologically dependent parallel evolution of male harm and female resistance in Drosophila melanogaster. Ecol Lett 17: 221-228.

Arnqvist G. 1994. The cost of male secondary sexual traits: Developmental constraints during ontogeny in a sexually dimorphic water strider. Am Nat 144: 119-132.

Arnqvist G. 2004. Sexual conflict and sexual selection: Lost in the chase. Evolution (NY) 58: 1383-1388.

Arnqvist G. 2006. Sensory exploitation and sexual conflict. Phil Trans R Soc 361: 375-386.

Arnqvist G, Nilsson T. 2000. The evolution of polyandry: Multiple mating and female fitness in insects. Anim Behav 60: 145-164. 
J.C. Perry and L. Rowe

Arnqvist G, Rowe L. 2002a. Comparative analysis unveils antagonistic coevolution between the sexes in a group of insects. Nature 415: 787-789.

Arnqvist G, Rowe L. 2002b. Correlated evolution of male and female morphologies in water striders. Evolution (NY) 56: 936-947.

Arnqvist G, Rowe L. 2005. Sexual conflict, Princeton University Press, Princeton, NJ.

Arnqvist G, Edvardsson M, Friberg U, Nilsson T. 2000. Sexual conflict promotes speciation in insects. Proc Nat Acad Sci 97: 10460-10464.

Badcock C, Crespi B. 2008. Battle of the sexes may set the brain. Nature 454: 1054-1055.

Bergsten J, Miller KB. 2007. Phylogeny of diving beetles reveals a coevolutionary arms race between the sexes. PLoS ONE 2: e522.

Bergsten J, Töyrä A, Nilsson AN. 2001. Intraspecific variation and intersexual correlation in secondary sexual characters of three diving beetles (Coleoptera: Dytiscidae). Biol J Linn Soc Lond 73: 221-232.

Bonduriansky R, Maklakov A, Zajitschek F, Brooks R. 2008. Sexual selection, sexual conflict and the evolution of ageing and life span. Func Ecol 22: 443-453.

Brommer JE, Fricke C, Edward DA, Chapman T. 2012. Interactions between genotype and sexual conflict environment influence transgenerational fitness in Drosophila melanogaster. Evolution (NY) 66: 517-531.

Carazo P, Tan CKW, Allen F, Wigby S, Pizzari T. 2014. Within-group male relatedness reduces harm to females in Drosophila. Nature 505: 672-675.

Chang AT, Sih A. 2013. Multilevel selection and effects of keystone hyperaggressive males on mating success and behavior in stream water striders. Behav Ecol 24: 11661176.

Chapman TW. 2006. Evolutionary conflicts of interest between males and females. Curr Biol 16: R744-R754.

Chapman T. 2008. The soup in my fly: Evolution, form and function of seminal fluid proteins. PLoS Biol 6: e179.

Chapman T, Arnqvist G, Bangham J, Rowe L. 2003a. Response to Eberhard and Cordero, and Córdoba-Aguilar and Contreras-Garduño: Sexual conflict and female choice. Trends Ecol Evol 18: 440-441.

Chapman T, Bangham J, Vinti G, Seifried B, Lung O, Wolfner MF, Smith HK, Partridge L. 2003b. The sex peptide of Drosophila melanogaster: Female post-mating responses analyzed by using RNA interference. Proc Natl Acad Sci 100: 9923-9928.

Cordero C, Eberhard WG. 2003. Female choice of sexually antagonistic male adaptations: A critical review of some current research. J Evol Biol 16: 1-6.

Crudgington HS, Siva-Jothy MT. 2000. Genital damage, kicking and early death. Nature 407: 855-856.

Crudgington HS, Beckerman AP, Brüstle L, Green K, Snook RR. 2005. Experimental removal and elevation of sexual selection: Does sexual selection generate manipulative males and resistant females? Am Nat 165: S72-S87.

Dickson BJ. 2008. Wired for sex: The neurobiology of Drosophila mating decisions. Science 322: 904-909.
Dougherty LR, Burdfield-Steel ER, Shuker DM. 2013. Sexual stereotypes: The case of sexual cannibalism. Anim Behav 85: 313-322.

Eberhard WG. 1985. Sexual selection and animal genitalia, Harvard University Press, Cambridge, MA.

Eberhard WG. 1997. Sexual selection by cryptic female choice in insects and arachnids. In The evolution of mating systems in insects and arachnids (ed. Choe JC, Crespi BJ), pp. 32-57. Cambridge University Press, Cambridge.

Eberhard WG. 2004a. Male-female conflict and genitalia: Failure to confirm predictions in insects and spiders. Biol Rev 79: $121-186$.

Eberhard WG. 2004b. Rapid divergent evolution of sexual morphology: Comparative tests of antagonistic coevolution and traditional female choice. Evolution (NY) 58: 1947-1970.

Eberhard WG. 2006. Sexually antagonistic coevolution in insects is associated with only limited morphological diversity. J Evol Biol 19: 657-681.

Eberhard WG. 2010. Evolution of genitalia: Theories, evidence, and new directions. Genetica 138: 5-18.

Eberhard WG, Cordero C. 2003. Sexual conflict and female choice. Trends Ecol Evol 18: 438-439.

Edvardsson M, Tregenza T. 2005. Why do male Callosobruchus maculatus harm their mates? Behav Ecol 16: 788793.

Eldakar OT, Dlugos MJ, Pepper JW, Wilson DS. 2009a. Aggressive mating as a tragedy of the commons in the water strider Aquarius remigis. Behav Ecol Soc 64: 25-33.

Eldakar OT, Dlugos MJ, Pepper JW, Wilson DS. 2009b. Population structure mediates sexual conflict in water striders. Science 326: 816.

Findlay GD, Swanson WJ. 2010. Proteomics enhances evolutionary and functional analysis of reproductive proteins. BioEssays 32: 26-36.

Fiumera AC, Dumont BL, Clark AG. 2006. Natural variation in male-induced "cost-of-mating" and allele-specific association with male reproductive genes in Drosophila melanogaster. Phil Trans R Soc 361: 355-361.

Friberg U, Arnqvist G. 2003. Fitness effects of female mate choice: Preferred males are detrimental for Drosophila melanogaster females. J Evol Biol 16: 797-811.

Fricke C, Perry J, Chapman T, Rowe L. 2009. The conditional economics of sexual conflict. Biol Lett 5: 671-674.

Fricke C, Andersson C, Arnqvist G. 2010a. Natural selection hampers divergence of reproductive traits in a seed beetle. J Evol Biol 23: 1857-1867.

Fricke C, Bretman A, Chapman T. 2010b. Female nutritional status determines the magnitude and sign of responses to a male ejaculate signal in Drosophila melanogaster. J Evol Biol 23: $157-165$.

Fricke C, Green D, Mills WE, Chapman T. 2013. Age-dependent female responses to a male ejaculate signal alter demographic opportunities for selection. Proc $R$ Soc $B$ 280: 20130428.

Friesen CR, Uhrig EJ, Squire MK, Mason RT, Brennan PL. 2014. Sexual conflict over mating in red-sided garter snakes (Thamnophis sirtalis) as indicated by experimental manipulation of genitalia. Proc $R$ Soc $B$ 281: 20132694. 
Gagnon M-C, Turgeon J. 2011. Sexual conflict in Gerris gillettei (Insecta: Hemiptera): Intraspecific intersexual correlated morphology and experimental assessment of behaviour and fitness. J Evol Biol 24: 1505-1516.

Gasparini C, Devigili A, Pilastro A. 2012. Cross-generational effects of sexual harassment on female fitness in the guppy. Evolution (NY) 66: 532-543.

Gavrilets S. 2000. Rapid evolution of reproductive barriers driven by sexual conflict. Nature 403: 886-889.

Gavrilets S, Hayashi TI. 2006. The dynamics of two- and three-way sexual conflicts over mating. Phil Trans $R$ Soc 361: 345-354.

Gavrilets S, Waxman D. 2002. Sympatric speciation by sexual conflict. Proc Natl Acad Sci 99: 10533-10538.

Gavrilets S, Arnqvist G, Friberg U. 2001. The evolution of female mate choice by sexual conflict. Proc R Soc B 268: 531-539.

Gershman SN, Hunt J, Sakaluk SK. 2013. Food fight: Sexual conflict over free amino acids in the nuptial gifts of male decorated crickets. J Evol Biol 26: 693-704.

Green KK, Madjidian JA. 2011. Active males, reactive females: Stereotypic sex roles in sexual conflict research? Anim Behav 81: 901-907.

Green KK, Kovalev A, Svensson EI, Gorb SN. 2013. Male clasping ability, female polymorphism and sexual conflict: Fine-scale elytral morphology as a sexually antagonistic adaptation in female diving beetles. $J R$ Soc Interface $\mathbf{1 0 .}$

Gwynne DT. 2008. Sexual conflict over nuptial gifts in insects. Annu Rev Entomol 53: 83-101.

Han CS, Jablonski PG. 2009. Female genitalia concealment promotes intimate male courtship in a water strider. PLoS ONE 4: e5793.

Han CS, Jablonski PG, Kim B, Park FC. 2010. Size-assortative mating and sexual size dimorphism are predictable from simple mechanics of mate-grasping behavior. $B M C$ Evol Biol 10: 359.

Härdling R, Bergsten J. 2006. Nonrandom mating preserves intrasexual polymorphism and stops population differentiation in sexual conflict. Am Nat 167: 401-409.

Härdling R, Smith HG. 2005. Antagonistic coevolution under sexual conflict. Evol Ecol 19: 137-150.

Hayashi TI, Vose M, Gavrilets S. 2007. Genetic differentiation by sexual conflict. Evolution (NY) 61: 516-529.

Holland B, Rice WR. 1998. Perspective: Chase-away sexual selection: Antagonistic seduction versus resistance. Evolution (NY) 52: 1-7.

Holland B, Rice WR. 1999. Experimental removal of sexual selection reverses intersexual antagonistic coevolution and removes a reproductive load. Proc Natl Acad Sci 96: 5083-5088.

Hotzy C, Arnqvist G. 2009. Sperm competition favors harmful males in seed beetles. Curr Biol 19: 404-407.

Hotzy C, Polak M, Ronn JL, Arnqvist G. 2012. Phenotypic engineering unveils the function of genital morphology. Curr Biol 22: 2258-2261.

Jiang PP, Bedhomme S, Prasad NG, Chippindale AK. 2011. Sperm competition and mate harm unresponsive to male-limited selection in Drosophila: An evolving genetic
The Evolution of Sexually Antagonistic Phenotypes

architecture under domestication. Evolution (NY) 65: 2448-2460.

Johnstone RA, Keller L. 2000. How males can gain by harming their mates: Sexual conflict, seminal toxins, and the cost of mating. Am Nat 156: 368-377.

Kazancioğlu E, Alonzo SH. 2012. The evolution of optimal female mating rate changes the coevolutionary dynamics of female resistance and male persistence. Phil Trans $R$ Soc 367: 2339-2347.

Khila A, Abouheif E, Rowe L. 2012. Function, developmental genetics, and fitness consequences of a sexually antagonistic trait. Science 336: 585-589.

Kimura M, Ihara Y. 2009. Replicator-dynamics models of sexual conflict. J Theor Biol 260: 90-97.

King EDA, Banks PB, Brooks RC. 2013. Sexual conflict in mammals: Consequences for mating systems and life history. Mamm Rev 43: 47-58.

Kirkpatrick M, Ryan MJ. 1991. The evolution of mating preferences and the paradox of the lek. Nature 350: 3338.

Kokko H, Brooks R. 2003. Sexy to die for? Sexual selection and the risk of extinction. Ann Zool Fennici 40: 207-219.

Kokko H, Brooks R, Jennions M, Morley J. 2003. The evolution of mate choice and mating biases. Proc R Soc B 270: 653-664.

Kokko H, Booksmythe I, Jennions MD. 2013. Causality and sex roles: Prejudice against patterns? A reply to Ah-King. Trends Ecol Evol 28: 2-4.

Kwan L, Cheng YY, Rodd FH, Rowe L. 2013. Sexual conflict and the function of genitalic claws in guppies (Poecilia reticulata). Biol Lett 9: 20130267.

Lande R. 1981. Models of speciation by sexual selection on plolygenic traits. Proc Natl Acad Sci 78: 3721-3725.

Lange R, Reinhardt K, Michiels NK, Anthes N. 2013. Functions, diversity, and evolution of traumatic mating. Biol Rev Camb Phil Soc 88: 585-601.

Lessells CM. 2006. The evolutionary outcome of sexual conflict. Phil Trans R Soc 361: 301-317.

Lew TA, Morrow EH, Rice WR. 2006. Standing genetic variance for female resistance to harm from males and its relationship to intralocus sexual conflict. Evolution (NY) 60: $97-105$.

Linder JE, Rice WR. 2005. Natural selection and genetic variation for female resistance to harm from males. $J$ Evol Biol 18: 568-575.

Long TAF, Pischedda A, Stewart AD, Rice WR. 2009. A cost of sexual attractiveness to high-fitness females. PLoS Biol 7: e1000254.

Madjidian JA, Green KK. 2012. A reply to Perry \& Rowe: Costs in sexual conflict research. Anim Behav 83: e14-e16.

Maklakov AA, Arnqvist G. 2009. Testing for direct and indirect effects of mate choice by manipulating female choosiness. Curr Biol 19: 1903-1906.

Martin OY, Hosken DJ. 2003. The evolution of reproductive isolation through sexual conflict. Nature 423: 979-982.

Martin OY, Hosken DJ. 2004. Reproductive consequences of population divergence through sexual conflict. Curr Biol 14: $906-910$. 
J.C. Perry and L. Rowe

Morrow EH, Arnqvist G. 2003. Costly traumatic insemination and a female counter-adaptation in bed bugs. Proc $R$ Soc B 270: 2377-2381.

Morrow EH, Connallon T. 2013. Implications of sex-specific selection for the genetic basis of disease. Evol Appl 6: 1208-1217.

Morrow EH, Arnqvist G, Pitnick S. 2003. Adaptation versus pleiotropy: Why do males harm their mates? Behav Ecol 14: $802-806$.

Nandy B, Gupta V, Sen S, Udaykumar N, Samant MA, Ali SZ, Prasad NG. 2013. Evolution of mate-harm, longevity and behaviour in male fruit flies subjected to different levels of interlocus conflict. BMC Evol Biol 13: 212.

Parker GA. 1979. Sexual selection and sexual conflict. In Sexual selection and reproductive competition in insects (ed. Blum MS, Blum NA), pp. 123-166. Academic, New York.

Parker GA. 2006. Sexual conflict over mating and fertilization: An overview. Phil Trans R Soc 361: 235-259.

Parker GA, Simmons LW. 1989. Nuptial feeding in insects: Theoretical models of male and female interests. Ethology 82: 3-26.

Perry JC, Rowe L. 2012a. Sex role stereotyping and sexual conflict theory. Anim Behav 83: e10-e13.

Perry JC, Rowe L. 2012b. Sexual conflict and antagonistic coevolution across water strider populations. Evolution 66: $544-557$.

Perry JC, Sirot L, Wigby S. 2013. The seminal symphony: How to compose an ejaculate. Trends Ecol Evol 28: 414422.

Pitnick S, Miller GT, Reagan J, Holland B. 2001. Males' evolutionary responses to experimental removal of sexual selection. Proc R Soc B 268: 1071-1080.

Pizzari T, Snook RR. 2003. Perspective: Sexual conflict and sexual selection: Chasing away paradigm shifts. Evolution (NY) 57: 1223-1236.

Plesnar-Bielak A, Skrzynecka AM, Prokop ZM, Kolasa M, Dzialo M, Radwan J. 2013. No evidence for reproductive isolation through sexual conflict in the bulb mite Rhizoglyphus robini. PLoS ONE 8: e74971.

Promislow DEL. 2003. Mate choice, sexual conflict and evolution of senescence. Behav Genet 33: 191-201.

Rankin DJ. 2011. Kin selection and the evolution of sexual conflict. J Evol Biol 24: 71-81.

Reinhardt K, Naylor R, Siva-Jothy MT. 2003. Reducing a cost of traumatic insemination: Female bedbugs evolve a unique organ. Proc R Soc B 270: 2371-2375.

Rice WR. 1996. Sexually antagonistic male adaptation triggered by experimental arrest of female evolution. Nature 381: $232-234$.

Rice WR, Linder JE, Friberg U, Lew TA, Morrow EH, Stewart AD. 2005. Inter-locus antagonistic coevolution as an engine of speciation: Assessment with hemiclonal analysis. Proc Natl Acad Sci 102: 6527-6534.

Rice WR, Stewart AD, Morrow EH, Linder JE, Orteiza N, Byrne PG. 2006. Assessing sexual conflict in the Drosophila melanogaster laboratory model system. Phil Trans $R$ Soc 361: 287-299.
Rogina B. 2009. The effect of sex peptide and calorie intake on fecundity in female Drosophila melanogaster. Scientific-WorldJournal 9: 1178-1189.

Rönn J, Katvala M, Arnqvist G. 2007. Coevolution between harmful male genitalia and female resistance in seed beetles. Proc Natl Acad Sci 104: 10921-10925.

Ronkainen K, Kaitala A, Huttunen R. 2005. The effect of abdominal spines on female mating frequency and fecundity in a water strider. J Insect Behav 18: 619-631.

Rowe L. 1994. The cost of mating and mate choice in water striders. Anim Behav 48: 1049-1056.

Rowe L, Arnqvist G. 2002. Sexually antagonistic coevolution in a mating system: Combining experimental and comparative approaches to address evolutionary processes. Evolution (NY) 56: 754-767.

Rowe L, Arnqvist G. 2012. Sexual selection and the evolution of genital shape and complexity in water striders. Evolution (NY) 66: 40-54.

Rowe L, Day T. 2006. Detecting sexual conflict and sexually antagonistic coevolution. Phil Trans $R$ Soc 361: 277-285.

Rowe L, Arnqvist G, Sih A, Krupa JJ. 1994. Sexual conflict and the evolutionary ecology of mating patterns: Water striders as a model system. Trends Ecol Evol 9: 289293.

Rowe L, Cameron E, Day T. 2005. Escalation, retreat, and female indifference as alternative outcomes of sexually antagonistic coevolution. Am Nat 165: S5-S18.

Rowe L, Westlake KP, Currie DC. 2006. Functional significance of elaborate secondary sexual traits and their evolution in the water strider genus Rheumatobates. Can Entomol 138: 568-577.

Schärer L, Rowe L, Arnqvist G. 2012. Anisogamy, chance and the evolution of sex roles. Trends Ecol Evol 27: $260-264$.

* Schärer L, Janicke T, Ramm SA. 2014. Sexual conflict in hermaphrodites. Cold Spring Harb Perspet Biol doi: 10.1101/cshperspect.a017673.

* Schneider JM. 2014. Sexual cannibalism as a manifestation of sexual conflict. Cold Spring Harb Perspet Biol doi: 10.1101/cshperspect.a017731.

Sih A, Watters JV. 2005. The mix matters: Behavioural types and group dynamics in water striders. Behaviour 142: 1417-1431.

Simmons LW, Parker GA. 1989. Nuptial feeding in insects: mating effort versus paternal investment. Ethology 81: $332-343$.

Stutt AD, Siva-Jothy MT. 2001. Traumatic insemination and sexual conflict in the bed bug Cimex lectularius. Proc Natl Acad Sci 98: 5683-5687.

Tatarnic NJ, Cassis G. 2010. Sexual coevolution in the traumatically inseminating plant bug genus Coridromius. $J$ Evol Biol 23: 1321-1326.

Tatarnic NJ, Cassis G, Hochuli DF. 2006. Traumatic insemination in the plant bug genus Coridromius Signoret (Heteroptera: Miridae). Biol Lett 2: 58-61.

Tatarnic NJ, Cassis G, Siva-Jothy MT. 2014. Traumatic insemination in terrestrial arthropods. Annu Rev Entomol 59: $245-261$. 
The Evolution of Sexually Antagonistic Phenotypes

Tregenza T, Wedell N, Chapman TW. 2006. Introduction. Sexual conflict: A new paradigm? Phil Trans R Soc 361: 229-234.

Usinger RL. 1966. Monograph of cimicidae (Hemiptera, Heteroptera). Entomological Society of America, College Park, MD.

Vahed K. 2007. All that glisters is not gold: Sensory bias, sexual conflict and nuptial feeding in insects and spiders. Ethology 113: 105-127.

Wigby S, Chapman TW. 2004. Female resistance to male harm evolves in response to manipulation of sexual conflict. Evolution (NY) 58: 1028-1037.
Wigby S, Chapman T. 2005. Sex peptide causes mating costs in female Drosophila melanogaster. Curr Biol 15: 316321.

Wolfner MF. 2002. The gifts that keep on giving: physiological functions and evolutionary dynamics of male seminal proteins in Drosophila. Heredity 88: 85-93.

Yamane T. 2013. Intra-specific variation in the effect of male seminal substances on female oviposition and longevity in Callosobruchus chinensis. Evol Biol 40: 133-140.

Zeh JA, Zeh DW. 2003. Toward a new sexual selection paradigm: Polyandry, conflict and incompatibility. Ethology 109: 929-950. 


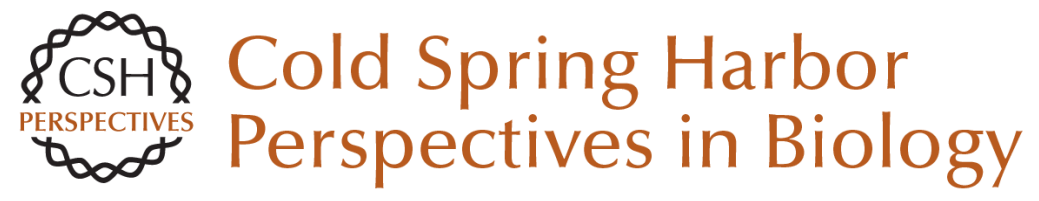

\section{The Evolution of Sexually Antagonistic Phenotypes}

Jennifer C. Perry and Locke Rowe

Cold Spring Harb Perspect Biol 2015; doi: 10.1101/cshperspect.a017558

Subject Collection The Genetics and Biology of Sexual Conflict

Mechanisms and Evidence of Genital

Coevolution: The Roles of Natural Selection, Mate Choice, and Sexual Conflict

Patricia L.R. Brennan and Richard O. Prum

The Evolution of Sexually Antagonistic

Phenotypes

Jennifer C. Perry and Locke Rowe

Reproductive Parasitism: Maternally Inherited

Symbionts in a Biparental World

Gregory D.D. Hurst and Crystal L. Frost

Sex-Biased Gene Expression and Sexual Conflict throughout Development

Fiona C. Ingleby, Ilona Flis and Edward H. Morrow

Human Homosexuality: A Paradigmatic Arena for

Sexually Antagonistic Selection?

Andrea Camperio Ciani, Umberto Battaglia and Giovanni Zanzotto

Sexual Conflict Arising from Extrapair Matings in Birds

Alexis S. Chaine, Robert Montgomerie and Bruce E. Lyon

Sexual Conflict and Seminal Fluid Proteins: A

Dynamic Landscape of Sexual Interactions

Laura K. Sirot, Alex Wong, Tracey Chapman, et al.

Conflict on the Sex Chromosomes: Cause, Effect, and Complexity

Judith E. Mank, David J. Hosken and Nina Wedell
Infanticide as Sexual Conflict: Coevolution of

Male Strategies and Female Counterstrategies Ryne A. Palombit

Copulatory Wounding and Traumatic

Insemination

Klaus Reinhardt, Nils Anthes and Rolanda Lange

Sexual Conflict in Hermaphrodites

Lukas Schärer, Tim Janicke and Steven A. Ramm

Sexual Conflict and Sperm Competition

Dominic A. Edward, Paula Stockley and David J. Hosken

Sexually Antagonistic Zygotic Drive: A New Form

of Genetic Conflict between the Sex

Chromosomes Urban Friberg and William R. Rice

Sex Chromosome Drive

Quentin Helleu, Pierre R. Gérard and Catherine Montchamp-Moreau

Is Sexual Conflict an "Engine of Speciation"? Sergey Gavrilets

Sexual Cannibalism as a Manifestation of Sexual Conflict

Jutta M. Schneider

For additional articles in this collection, see http://cshperspectives.cshlp.org/cgi/collection/

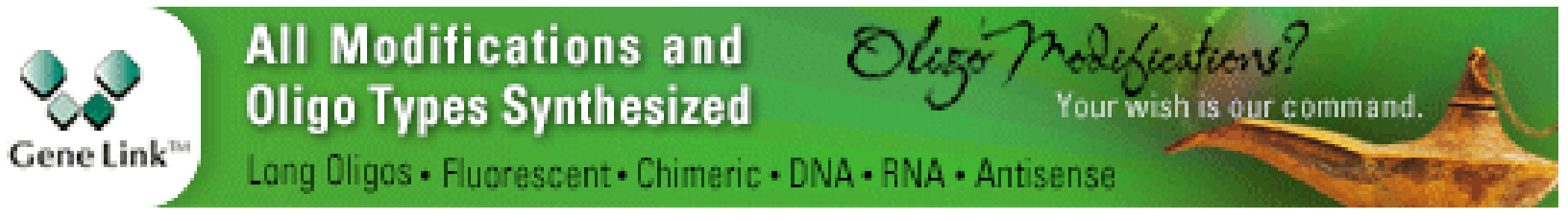

